\title{
Characteristics of 'foregut' carcinoid tumours occurring in multiple endocrine neoplasia type 1 (MEN1)
}

\section{Yang, K. Mann, J. Winceslaus, R. Khan, D. Berney, S. Akker, M. Waterhouse, W. Drake, M. Druce St Bartholomew's Hospital, London, UK.}

\section{Background}

The most common 'foregut' carcinoid tumours in MEN1 are pancreatic neuroendocrine tumours (pNETs). A number of other 'foregut' carcinoids may also occur in MEN1, including those of bronchial, thymic and gastrointestinal origin. These latter tumours have reported rates of prevalence ranging from $2 \%$ for thymic and bronchial carcinoids, to $10 \%$ for gastric carcinoids. Thymic carcinoids are known to be aggressive in nature, whereas bronchial carcinoids are thought to be mostly indolent with no effect on increased mortality in MEN1.

\section{Aims}

To evaluate the prevalence and characteristics of carcinoid tumours in current patients under follow up with MEN1 at a tertiary referral centre.

\section{Methods}

Data were collected from case notes, histopathology reports and cross-sectional imaging.

\section{Results}

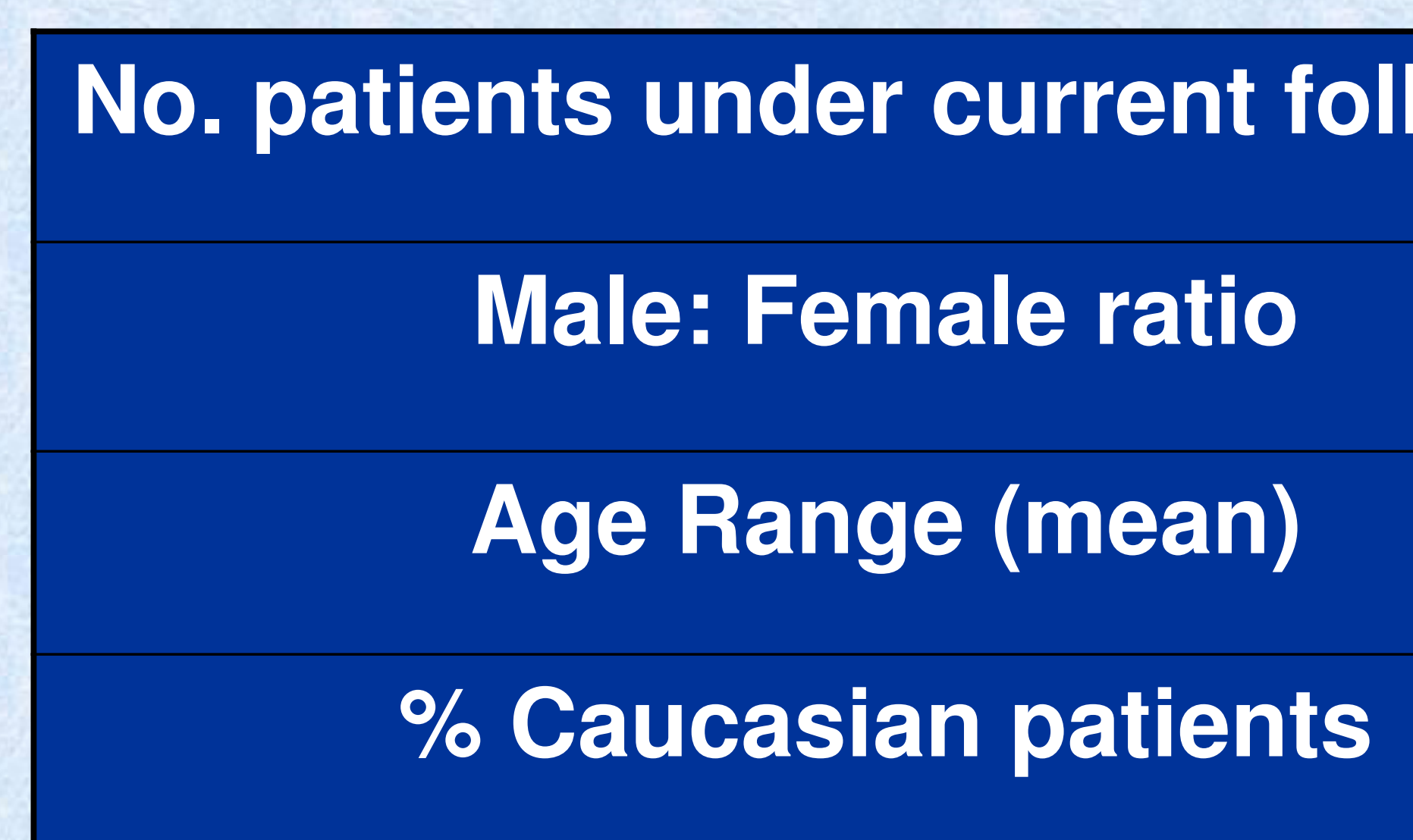

\begin{tabular}{|c|}
\hline 46 \\
\hline $17: 29$ \\
\hline $18-79$ yrs (53.7yrs) \\
\hline $89 \%$ \\
\hline
\end{tabular}

31 'foregut' carcinoids were identified in 27 patients

\begin{tabular}{|c|c|c|c|c|c|}
\hline non-pNET patients & $\begin{array}{c}\text { Age at diagnosis } \\
\text { (yrs) }\end{array}$ & Gender & $\begin{array}{c}\text { Concurrent } \\
\text { pNET }\end{array}$ & Pituitary Lesion & $\begin{array}{c}\text { Hyper- } \\
\text { parathyroidism }\end{array}$ \\
\hline (P1) Bronchial * & 36 & Female & None & non-functioning microadenoma & Present \\
\hline (P2 Bronchial ** & 71 & Female & Gastrinoma & None & Present \\
\hline (P3) Bronchial ** & 34 & Male & Pancreas tail lesion & None & Present \\
\hline (P4) Thymic & 56 & Male & Gastrinoma & None & Present \\
\hline (P5) Gastric & 38 & Male & None & None & Present \\
\hline (P6) Indeterminate & 46 & Male & Islet cell adenomas & Macroprolactinoma & Present \\
\hline
\end{tabular}

* secretory, ${ }^{* *}$ non-secretory

Of the bronchial carcinoids, $\mathrm{P} 1$ had a secretory tumour which was successfully resected, $\mathrm{P} 2$ and $\mathrm{P} 3$ had non-secretory tumours, both of whom showed aggressive malignant potential. P2 is deceased and P3 is currently undergoing adjuvant chemotherapy. P4 had successful excision of a thymic mass with no recurrence to date. P5 underwent a total gastrectomy following a GI bleed at diagnosis of gastric carcinoid. Histology from excision of a mediastinal mass in P6 showed a well differentiated atypical carcinoid with $\mathrm{Ki}-67$ up to $40 \%$ and pleural and vascular invasion.

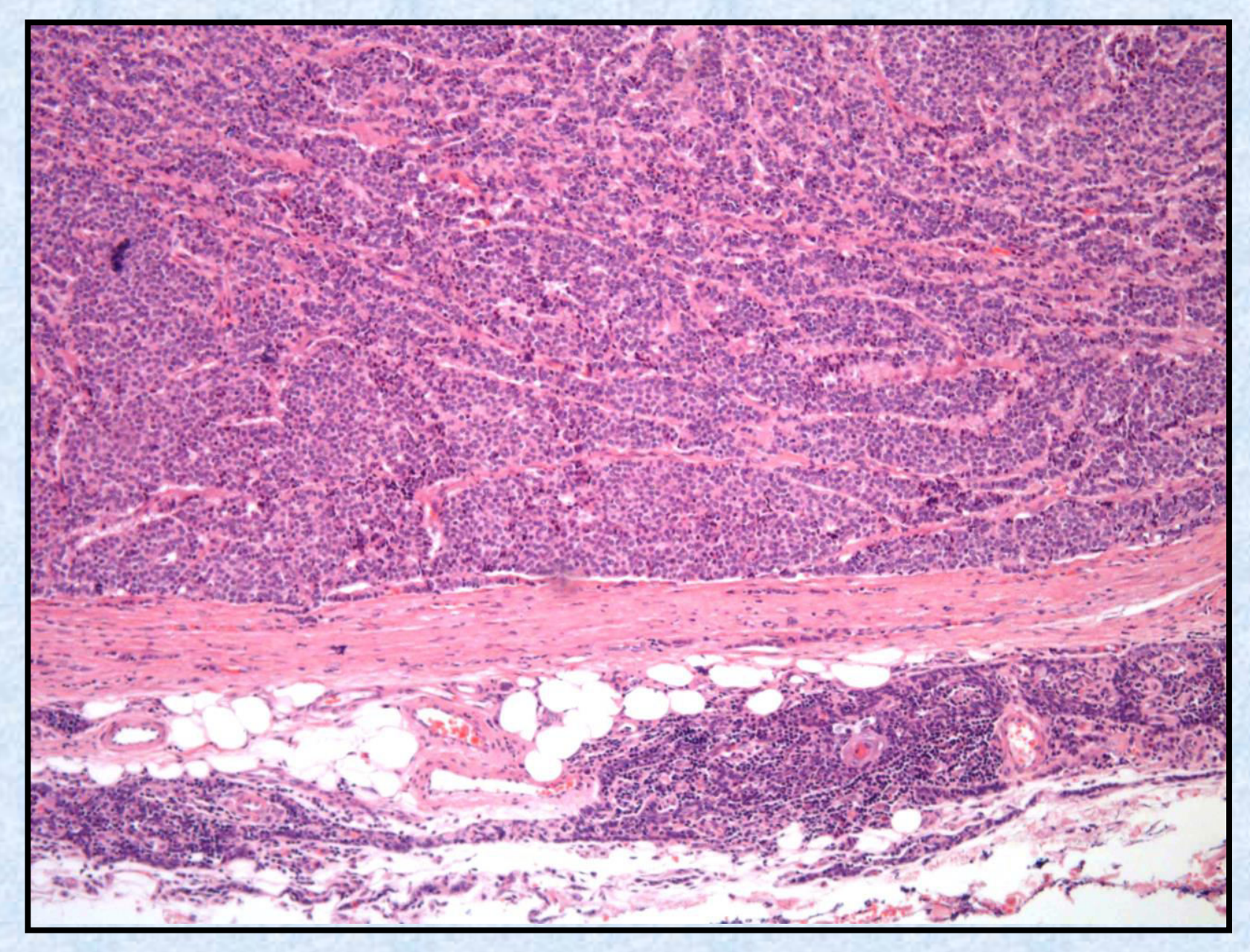

(1)

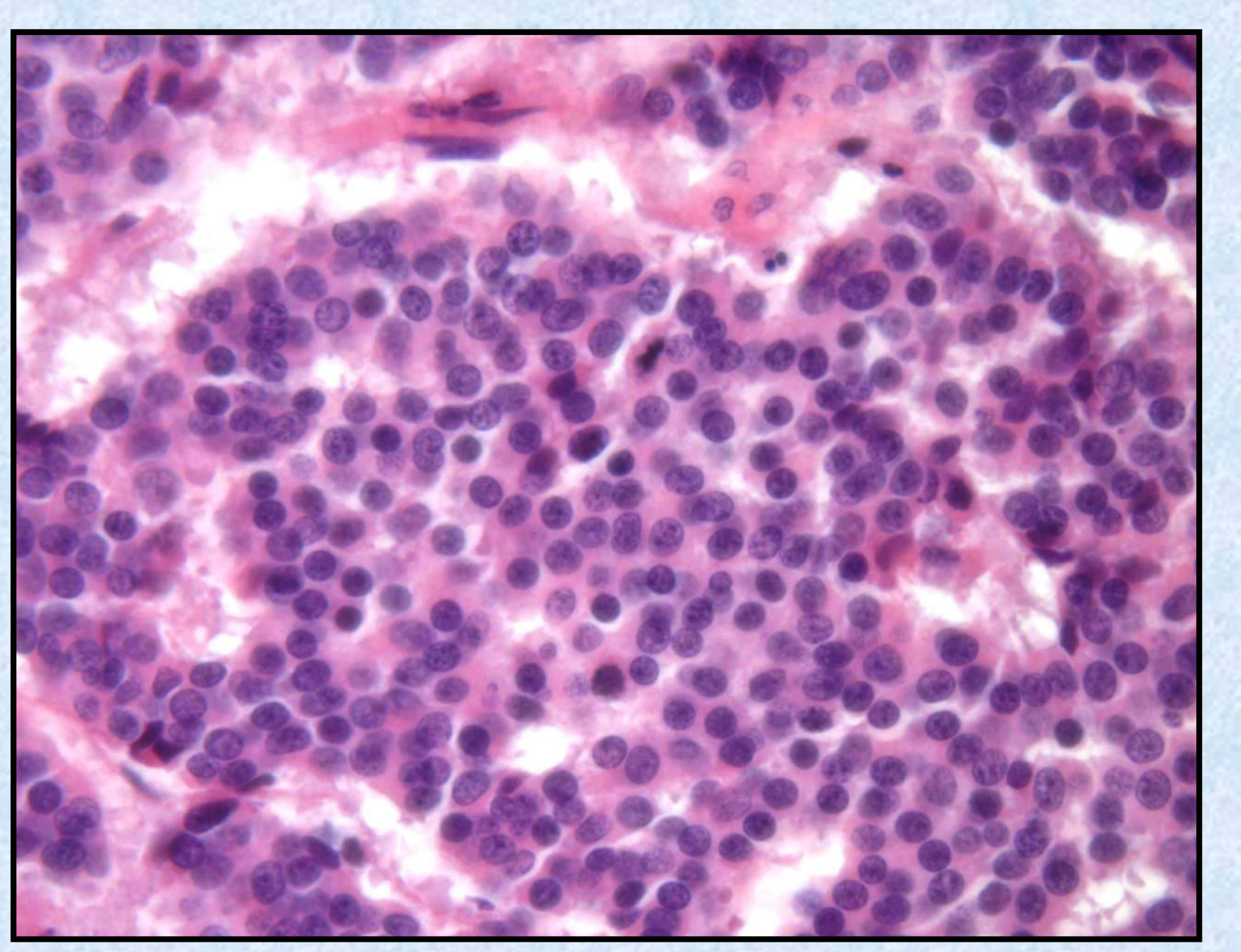

(2)

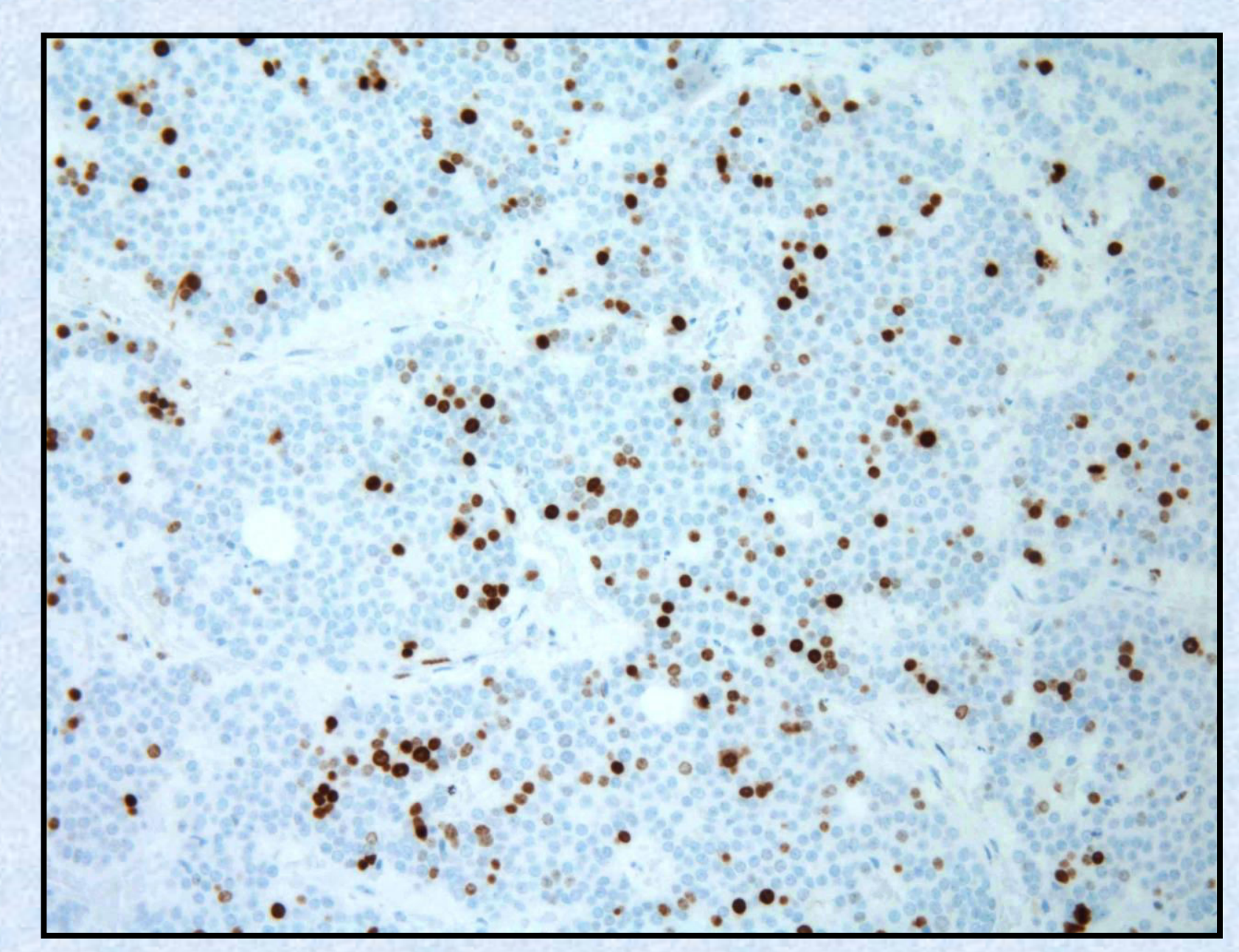

(3)
Histology slides from P3

1) Low power field showing neuroendocrine tissue within a circumscribed border with rim of compressed fibrous connective tissue.

2) High power field showing cells with morphology in keeping with atypical carcinoid tumour.

3) Immunohistochemistry staining with strong positivity for Chromogranin A and Ki-67 up to $30 \%$ in certain areas.

\section{Discussion}

In this contemporary series of non-related MEN1 patients, the prevalence of non-pancreatic 'foregut' carcinoid tumours is $13 \%$. This is higher than most reported series. In contrast to previous studies, non-secretory bronchial carcinoids showed aggressive characteristics in our patients. Current MEN1 consensus guidelines suggest 1-2 yearly surveillance imaging by CT or MRI to detect intrathoracic carcinoids, and prophylactic thymectomy is advocated to prevent occurrence of thymic malignancy. Further prospective studies would be useful in guiding decisions regarding prophylactic resection of asymptomatic pulmonary nodules. 